\title{
Tree-unitarity bounds for THDM Higgs masses revisited
}

\author{
J. Hořejší, M. Kladiva \\ Institute of Particle and Nuclear Physics, Faculty of Mathematics and Physics, \\ Charles University, V Holešovičkách 2, CZ-180 00 Prague 8, Czech Republic
}

October 12,2005

\begin{abstract}
We have reconsidered theoretical upper bounds on the scalar boson masses within the two-Higgs-doublet model (THDM), employing the well-known technical condition of tree-level unitarity. Our treatment provides a modest extension and generalization of some previous results of other authors. We present a rather detailed discussion of the solution of the relevant inequalities and offer some new analytic formulae as well as numerical values for the Higgs mass bounds in question. A comparison is made with the earlier results on the subject that can be found in the literature.
\end{abstract}

\section{Introduction}

The two-Higgs-doublet model (THDM) of electroweak interactions is one of the simplest extensions of the Standard Model (SM). It incorporates two complex scalar doublets in the Higgs sector, but otherwise its structure is the same as that of the SM. Obviously, such a theory is rather appealing on purely aesthetic grounds: in view of the familiar doublet pattern of the elementary fermion spectrum, one can speculate that an analogous organizational principle might work for the "scalar Higgs matter" as well. Further, any Higgs sector built upon doublets only is known to preserve naturally the famous lowestorder electroweak relation $\rho=1$ (where $\rho=m_{W}^{2} /\left(m_{Z}^{2} \cos ^{2} \theta_{W}\right)$ ), which has been tested with good accuracy. On the phenomenological side, an important aspect of the THDM is that its Higgs sector may provide an additional source of $C P$ violation; in fact, this was the primary motivation for introducing such a model in the early literature on spontaneously broken gauge theories in particle physics [1. Of course, there is at least one more reason why the THDM has become popular ${ }^{1}$ during the last two decades or so: its Higgs sector essentially coincides with that of the minimal supersymmetric SM (MSSM), but the values of the relevant parameters are less restricted. The spectrum of physical Higgs particles within

\footnotetext{
${ }^{1}$ For useful reviews of the subject see e.g. [2, 3]
} 
THDM consists of five scalar bosons, three of them being electrically neutral (denoted usually as $h, H$ and $A^{0}$ ) and the other two charged $\left(H^{ \pm}\right)$. At present, some partial information concerning direct experimental lower bounds for the Higgs masses is available, coming mostly from the LEP data (cf. 4).

On the other hand, it is also interesting to know what could be possible theoretical limitations for masses of the so far elusive Higgs particles within such a "quasi-realistic" model. For this purpose, some rather general methods have been invented, based mostly on the requirements of internal consistency of the quantum field theoretical description of the relevant physical quantities. One particular approach, which is perhaps most straightforward in this regard, relies on perturbative unitarity of the $S$-matrix. In its simplest form it is implemented at the lowest order, by imposing unitarity constraints on the tree-level amplitudes of a suitable set of scattering processes. Let us recall that this technique was originally developed by B.W. Lee, C. Quigg and H. Thacker (LQT), who employed it in their well-known analysis of perturbative upper bound for the SM Higgs boson mass 5 . The LQT method was subsequently applied also to electroweak models with extended Higgs sectors; some results can be found under refs. 6], 7], 8]. In particular, authors of the papers [7], 8] analyzed in this way a restricted version of the THDM with $C P$ conserving Higgs sector and obtained slightly differing values of the bounds in question (due to slightly different implementations of the LQT method). Recently, the issue of tree-unitarity constraints for THDM Higgs boson masses has been taken up again in the work [9] (see also [10, [11]), where a rather general model involving $C P$ violation has been considered; this seems to be another vindication of the persisting interest in the subject.

The purpose of the present paper is to supplement and extend the existing results concerning the THDM Higgs mass upper bounds. We carry out a rather detailed analysis of a relevant set of inequalities that follow from the requirement of tree-level unitarity. In particular, the procedure of explicit solution of these constraints is discussed in considerable detail and, among other things, some results of the corresponding numerical calculations within a general THDM are presented. For the model without $C P$ violation we were able to find a set of analytic expressions as well. Note that in this latter case, most of the calculational details are contained also in an earlier unpublished work by one of us (see [12]). Let us also remark that there is no substantial overlap of the material presented in [9, 10, 11] with our results, so we believe that it makes sense to offer our detailed analysis as a contribution to the current literature on the particular problem in question.

The plan of our paper is as follows: In Sect. 2 the THDM scalar potential and the scalar fields are described in some detail, in Sect. 3 we summarize briefly the LQT method and its implementation within THDM and in Sect. 4 the relevant inequalities expressing the tree-unitarity constraints are examined. The main analytic results for the mass bounds in question are contained in sections 5 , 囵 7 and Sect. 8 contains numerical results obtained in the $C P$-violating case (where we have not been able to find analytical results). The main results are summarized in Sect. 9 . 


\section{THDM scalar potential}

The most general scalar potential within THDM that is invariant under $S U(2) \times U(1)$ can be written as (cf. [13] or [3])

$$
\begin{gathered}
V(\Phi)=\lambda_{1}\left(\Phi_{1}^{\dagger} \Phi_{1}-\frac{v_{1}^{2}}{2}\right)^{2}+\lambda_{2}\left(\Phi_{2}^{\dagger} \Phi_{2}-\frac{v_{2}^{2}}{2}\right)^{2}+\lambda_{3}\left(\Phi_{1}^{\dagger} \Phi_{1}-\frac{v_{1}^{2}}{2}+\Phi_{2}^{\dagger} \Phi_{2}-\frac{v_{2}^{2}}{2}\right)^{2}+ \\
\lambda_{4}\left[\left(\Phi_{1}^{\dagger} \Phi_{1}\right)\left(\Phi_{2}^{\dagger} \Phi_{2}\right)-\left(\Phi_{1}^{\dagger} \Phi_{2}\right)\left(\Phi_{2}^{\dagger} \Phi_{1}\right)\right]+\lambda_{5}\left[\operatorname{Re}\left(\Phi_{1}^{\dagger} \Phi_{2}\right)-\frac{v_{1} v_{2}}{2} \cos \xi\right]^{2}+\lambda_{6}\left[\operatorname{Im}\left(\Phi_{1}^{\dagger} \Phi_{2}\right)-\frac{v_{1} v_{2}}{2} \sin \xi\right]^{2}
\end{gathered}
$$

Note that such a form involves $C P$ violation, which is due to $\xi \neq 0$ [3]. It also possesses an approximate discrete $Z_{2}$ symmetry under $\Phi_{2} \rightarrow-\Phi_{2}$; this is broken "softly", by means of the quadratic term

$$
v_{1} v_{2}\left(\lambda_{5} \cos \xi \operatorname{Re}\left(\Phi_{1}^{\dagger} \Phi_{2}\right)+\lambda_{6} \sin \xi \operatorname{Im}\left(\Phi_{1}^{\dagger} \Phi_{2}\right)\right)=v_{1} v_{2} \operatorname{Re}\left[\left(\lambda_{5} \cos \xi-i \lambda_{6} \sin \xi\right) \Phi_{1}^{\dagger} \Phi_{2}\right]
$$

Let us recall that the main purpose of such an extra partial symmetry within THDM is to suppress naturally the flavour-changing processes mediated by neutral scalar exchanges that could otherwise arise within the quark Yukawa sector 14. Note also that if such a symmetry were exact, there would be no $C P$ violation in the Higgs sector of the considered model. For further remarks concerning the role of the $Z_{2}$ symmetry see e.g. 9] and references therein. As a quantitative measure of the $Z_{2}$ violation we introduce a parameter $\nu$, defined as

$$
\nu=\sqrt{\lambda_{5}^{2} \cos ^{2} \xi+\lambda_{6}^{2} \sin ^{2} \xi}
$$

(note that our definition of the $\nu$ differs slightly from that used in [9].) The minimum of the potential (1) occurs at

$$
\Phi_{1}=\frac{1}{\sqrt{2}}\left(\begin{array}{c}
0 \\
v_{1}
\end{array}\right), \quad \Phi_{2}=\frac{1}{\sqrt{2}}\left(\begin{array}{c}
0 \\
v_{2}
\end{array}\right) \mathrm{e}^{i \xi}
$$

where we have adopted, for convenience, the usual simple choice of phases. Such a minimum determines vector boson masses through the Higgs mechanism; in particular, for the charged $W$ boson one gets $m_{W}^{2}=\frac{1}{2} g^{2}\left(v_{1}^{2}+v_{2}^{2}\right)$, with $g$ standing for $S U(2)$ coupling constant. In a standard notation one then writes $v_{1}=v \cos \beta, v_{2}=v \sin \beta$, where $v$ is the familiar electroweak scale, $v=\left(G_{F} \sqrt{2}\right)^{-1 / 2} \doteq 246 \mathrm{GeV}$ and $\beta$ is a free parameter. THDM involves eight independent scalar fields: three of them can be identified with the would-be Goldstone bosons $w^{ \pm}, z$ (the labelling is chosen so as to indicate that they are direct counterparts of the massive vector bosons $W^{ \pm}, Z$ within an $R$-gauge) and the remaining five correspond to physical Higgs particles - the charged $H^{ \pm}$and the neutral ones $h, H, A^{0}$.

We will now describe the above-mentioned Goldstone and Higgs bosons in more detail. To this end, let us start with a simple representation of the doublets, namely

$$
\Phi_{1}=\left(\begin{array}{c}
w_{1}^{-} \\
\frac{1}{\sqrt{2}}\left(v_{1}+h_{1}+i z_{1}\right)
\end{array}\right) \quad \Phi_{2}=\left(\begin{array}{c}
w_{2}^{-} \\
\frac{1}{\sqrt{2}}\left(\mathrm{e}^{i \xi} v_{2}+h_{2}+i z_{2}\right)
\end{array}\right)
$$


Of course, the scalar fields introduced in (5) are in general unphysical; the $w_{1,2}^{ \pm}$are taken to be complex and the remaining ones real, but otherwise arbitrary. Note that an advantage of such a parametrization is that the form of the quartic interactions is then the same as in $C P$ conserving case. The proper Goldstone and Higgs fields are found through a diagonalization of the quadratic part of the potential (11). When doing it, a convenient starting point is a slightly modified doublet parametrization

$$
\Phi_{1}=\left(\begin{array}{c}
w_{1}^{-} \\
\frac{1}{\sqrt{2}}\left(v_{1}+h_{1}+i z_{1}\right)
\end{array}\right) \quad \Phi_{2}=\left(\begin{array}{c}
w_{2}^{\prime-} \\
\frac{1}{\sqrt{2}}\left(v_{2}+h_{2}^{\prime}+i z_{2}^{\prime}\right)
\end{array}\right) \mathrm{e}^{i \xi}
$$

that is obtained from (5) by means of the unitary transformation $h_{2}^{\prime}=h_{2} \cos \xi+z_{2} \sin \xi$, $z_{2}^{\prime}=z_{2} \cos \xi-h_{2} \sin \xi$ a $w_{2}^{\prime \pm}=\mathrm{e}^{-i \xi} w_{2}^{ \pm}$. Next, the scalar fields in (6) are rotated pairwise as

$$
\begin{gathered}
\left(\begin{array}{l}
H^{\prime} \\
h^{\prime}
\end{array}\right)=\left(\begin{array}{cc}
\cos \beta & \sin \beta \\
-\sin \beta & \cos \beta
\end{array}\right)\left(\begin{array}{l}
h_{1} \\
h_{2}^{\prime}
\end{array}\right) \quad\left(\begin{array}{c}
A^{\prime} \\
z
\end{array}\right)=\left(\begin{array}{cc}
\cos \beta & \sin \beta \\
-\sin \beta & \cos \beta
\end{array}\right)\left(\begin{array}{l}
z_{1} \\
z_{2}^{\prime}
\end{array}\right) \\
\left(\begin{array}{c}
\zeta \\
w
\end{array}\right)=\left(\begin{array}{cc}
\cos \beta & \sin \beta \\
-\sin \beta & \cos \beta
\end{array}\right)\left(\begin{array}{l}
w_{1} \\
w_{2}^{\prime}
\end{array}\right)
\end{gathered}
$$

When the quadratic part of (II) is recast in terms of the new variables, one finds out that the $z, w^{ \pm}$are massless Goldstone bosons and the $H^{ \pm}$represent massive charged scalars. At this stage, the fields $h^{\prime}, H^{\prime}, A^{\prime}$ are still mixed and their mass matrix reads

$$
\frac{1}{2}\left(\begin{array}{ccc}
\mathrm{s}_{2 \beta}^{2}\left(\lambda_{1}+\lambda_{2}\right)+\mathrm{c}_{2 \beta}^{2}\left(\mathrm{c}_{\xi}^{2} \lambda_{5}+\mathrm{s}_{\xi}^{2} \lambda_{6}\right) & \mathrm{s}_{2 \beta}\left[-2 \mathrm{c}_{\beta}^{2} \lambda_{1}+2 \mathrm{~s}_{\beta}^{2} \lambda_{2}+\mathrm{c}_{2 \beta}\left(\mathrm{c}_{\xi}^{2} \lambda_{5}+\mathrm{s}_{\xi}^{2} \lambda_{6}\right)\right] & \frac{1}{2} \mathrm{c}_{2 \beta} \mathrm{s}_{2 \xi}\left(\lambda_{6}-\lambda_{5}\right) \\
\mathrm{s}_{2 \beta}\left[-2 \mathrm{c}_{\beta}^{2} \lambda_{1}+2 \mathrm{~s}_{\beta}^{2} \lambda_{2}+\mathrm{c}_{2 \beta}\left(\mathrm{c}_{\xi}^{2} \lambda_{5}+\mathrm{s}_{\xi}^{2} \lambda_{6}\right)\right] & 4\left[\mathrm{c}_{\beta}^{4} \lambda_{1}+\mathrm{s}_{\beta}^{4} \lambda_{2}+\lambda_{3}+\mathrm{c}_{\beta}^{2} \mathrm{~s}_{\beta}^{2}\left(\mathrm{c}_{\xi}^{2} \lambda_{5}+\mathrm{s}_{\xi}^{2} \lambda_{6}\right)\right] & \frac{1}{2} \mathrm{~s}_{2 \beta} \mathrm{s}_{2 \xi}\left(\lambda_{6}-\lambda_{5}\right) \\
\frac{1}{2} \mathrm{c}_{2 \beta} \mathrm{s}_{2 \xi}\left(\lambda_{6}-\lambda_{5}\right) & \frac{1}{2} \mathrm{~s}_{2 \beta} \mathrm{s}_{2 \xi}\left(\lambda_{5}-\lambda_{6}\right) & \mathrm{s}_{\xi}^{2} \lambda_{5}+\mathrm{c}_{\xi}^{2} \lambda_{6}
\end{array}\right)
$$

By diagonalizing it, one gets the true Higgs bosons $h, H, A^{0}$. The operation of charge conjugation $C$ means the complex conjugation of these physical fields (i.e. not of those appearing in the parametrization (5) ). However, we can employ the representation (6) involving fields that are linear combinations of real variables without complex coefficients. Note that for $\xi=0$ (the $C P$-conserving case) the $A$ is a $C P$-odd Higgs boson $\left(A^{\prime}=A\right.$ in such a case) and $H, h$ are $C P$ even. Such a statement is also true when $\xi=\pi / 2$ and/or $\lambda_{5}=\lambda_{6}$; as we shall see later in this section, for these particular values of parameters there is again no $C P$ violation in the potential (11).

For $\xi=0$ the Higgs boson masses can be calculated explicitly, and subsequently one can express the coupling constants $\lambda_{i}$ in terms of masses and a mixing angle defined through

$$
\left(\begin{array}{l}
h_{1} \\
h_{2}
\end{array}\right)=\left(\begin{array}{rr}
\cos \alpha & -\sin \alpha \\
\sin \alpha & \cos \alpha
\end{array}\right)\left(\begin{array}{l}
h \\
H
\end{array}\right)
$$

Let us now express the $\lambda_{1,2,3,4}$ in terms of the Higgs boson masses in the case $\xi=0$ (as we 
have only four distinct masses, we leave the $\lambda_{5}$ as a free parameter). One gets

$$
\begin{aligned}
& \lambda_{4}=2 v^{-2} m_{ \pm}^{2} \quad \lambda_{6}=2 v^{-2} m_{A}^{2} \quad \lambda_{3}=2 v^{-2} \frac{s_{\alpha} c_{\beta}}{s_{\beta} c_{\beta}}\left(m_{H}^{2}-m_{h}^{2}\right)-\frac{\lambda_{5}}{4} \\
& \lambda_{1}=\frac{1}{2} v^{-2}\left[c_{\alpha}^{2} m_{H}^{2}+s_{\alpha}^{2} m_{h}^{2}-\frac{s_{\alpha} c_{\beta}}{\tan \beta}\left(m_{H}^{2}-m_{h}^{2}\right)\right]-\frac{\lambda_{5}}{4}\left(\frac{1}{\tan ^{2} \beta}-1\right) \\
& \lambda_{2}=\frac{1}{2} v^{-2}\left[s_{\alpha}^{2} m_{H}^{2}+c_{\alpha}^{2} m_{h}^{2}-s_{\alpha} c_{\beta} \tan \beta\left(m_{H}^{2}-m_{h}^{2}\right)\right]-\frac{\lambda_{5}}{4}\left(\frac{1}{\tan ^{2} \beta}-1\right)
\end{aligned}
$$

Note also that the matrix of the quadratic form of the scalar fields is the Hessian of the potential at its minimum. The condition for the existence of a minimum is that the Hessian is positive definite, and this in turn means that the Higgs boson masses (squared) are positive.

Finally, let us discuss briefly the particular cases $\xi=0, \xi=\pi / 2$ and $\lambda_{5}=\lambda_{6}$. The case $\xi=0$ represents a model without $C P$ violation within the scalar sector, as it is described in [3]. The case $\xi=\pi / 2$ can be analyzed easily in the parametrization (6); using this, the potential can be viewed as the case $\xi=0$ with the change of notation

$$
\Phi_{1}^{\prime}=\Phi_{1} \quad \Phi_{2}^{\prime}=i \Phi_{2} \quad \lambda_{5} \leftrightarrow \lambda_{6}
$$

Thus, the two cases are equivalent. When $\lambda_{6}=\lambda_{5}$, the $\xi$-dependent part of the potential can be recast as

$$
\lambda_{5}\left(\operatorname{Re}\left(\Phi_{1}^{\dagger} \Phi_{2}\right)-\frac{v_{1} v_{2}}{2} \cos \xi\right)^{2}+\lambda_{6}\left(\operatorname{Im}\left(\Phi_{1}^{\dagger} \Phi_{2}\right)-\frac{v_{1} v_{2}}{2} \sin \xi\right)^{2}=\lambda_{6}\left|\Phi_{1}^{\dagger} \Phi_{2}-\frac{v_{1} v_{2}}{2} e^{i \xi}\right|^{2}
$$

The remaining terms do not depend on the relative phase between $\Phi_{1}$ and $\Phi_{2}$, so that the phase factor $\mathrm{e}^{i \xi}$ can be transformed away and one thus again has a $C P$-conserving case. A particular consequence of such an analysis is that for $\nu=0$ there can be no $C P$ violation.

\section{$3 \quad$ LQT method}

For finding the upper bounds on the Higgs boson masses we will employ the well-known LQT method invented three decades ago [5. This method relies on imposing the condition of perturbative (in particular, tree-level) unitarity on an appropriate set of physical scattering processes. Within a renormalizable theory, the scattering amplitudes are "asymptotically flat", i.e. they do not exhibit any power-like growth in the high-energy limit. However, the dominant couplings are typically proportional to the scalar boson masses and one can thus obtain useful technical constraints on their values. In the pioneering paper [5] the method was applied to the minimal SM, and several groups of authors employed it subsequently within models involving an extended Higgs sector, in particular the THDM (cf. [6], [7], [8]). The results of various authors differ slightly, so it perhaps makes sense to reconsider the corresponding calculation and present, for the sake of clarity, some additional technical details of the whole procedure. 
In the spirit of the LQT approach, our analysis is based on the condition of tree-level $S$-matrix unitarity within the subspace of two-particle states. Instead of the unitarity condition used in the original paper [5], we can adopt an improved constraint for the $s$-wave partial amplitude $\mathcal{M}_{0}$, namely

$$
\left|\operatorname{Re} \mathcal{M}_{0}\right| \leq \frac{1}{2}
$$

(cf. [15). Note that the tree-level matrix elements in question are real, and in the highenergy limit their leading contributions do not involve any angular dependence. Thus, the $\mathcal{M}_{0}$ generally coincides with the full tree-level (asymptotic) matrix element $\mathcal{M}$, up to a conventional normalization factor of $16 \pi$ appearing in the standard partial-wave expansion. The effective unitarity constraint (14) then becomes

$$
|\mathcal{M}| \leq 8 \pi
$$

For an optimal implementation of the unitarity constraints we will consider the eigenvalues of the matrix $M_{i j}=\mathcal{M}_{i \rightarrow j}$ where the indices $i$ and $j$ label symbolically all possible twoparticle states. Having in mind our primary goal, we take into account only binary processes whose matrix elements involve the Higgs boson masses in the leading order, in particular in the $O\left(E^{0}\right)$ terms. Invoking arguments analogous to those used in the original paper [5], one can show that the relevant contributions descend from the interactions of Higgs scalars and longitudinal vector bosons. Using the equivalence theorem for longitudinal vector bosons and Goldstone bosons (see e.g. [5], [16]) one finds out, in accordance with the LQT treatment, that the only relevant contributions come from the amplitudes involving Higgs bosons and unphysical Goldstone bosons (that occur in an $R$-gauge formulation of the theory). It means that we will examine the above-mentioned matrix $M_{i j}$, including all two-particle states made of the scalars (both physical and unphysical) $w^{ \pm}, z, H^{ \pm}, A^{0}, H, h$. It is not difficult to see that the leading terms in the individual amplitudes are determined by the direct (contact) quartic scalar interactions, while the triple vertices enter second order Feynman graphs and their contributions are suppressed by the propagator effects in the high energy expansion.

As noted above, we will be mainly concerned with the eigenvalues of the two-particle scattering matrix. It means that for our purpose we can consider, equivalently, any unitary transformation of the matrix $M_{i j}$. In particular, it is more convenient to take, instead of the $M_{i j}$, a matrix consisting of the scattering amplitudes between the two-particle states made of the "particles" $w_{a}^{ \pm}, z_{a}, h_{a}$ corresponding to the parametrization (15). The eigenvalues of this matrix can be found in the earlier paper [8].

Matrix elements for the scattering processes corresponding to the two-particle states 
$\left(w_{1}^{+} w_{2}^{-}, w_{2}^{+} w_{1}^{-}, h_{1} z_{2}, h_{2} z_{1}, z_{1} z_{2}, h_{1} h_{2}\right)$ form the submatrix

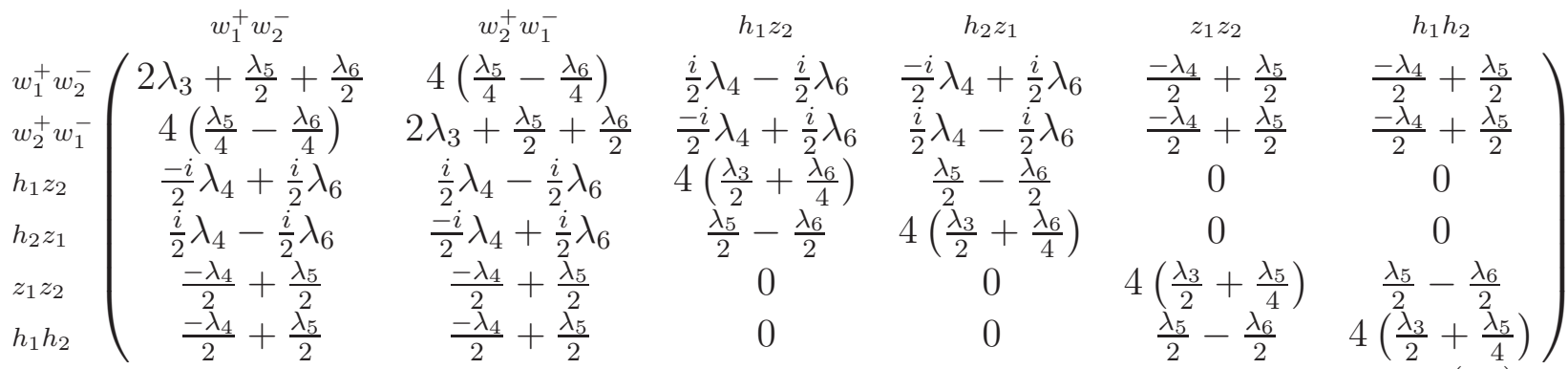

with eigenvalues

$$
\begin{aligned}
& e_{1}=2 \lambda_{3}-\lambda_{4}-\frac{1}{2} \lambda_{5}+\frac{5}{2} \lambda_{6} \\
& e_{2}=2 \lambda_{3}+\lambda_{4}-\frac{1}{2} \lambda_{5}+\frac{1}{2} \lambda_{6} \\
& f_{+}=2 \lambda_{3}-\lambda_{4}+\frac{5}{2} \lambda_{5}-\frac{1}{2} \lambda_{6} \\
& f_{-}=2 \lambda_{3}+\lambda_{4}+\frac{1}{2} \lambda_{5}-\frac{1}{2} \lambda_{6} \\
& f_{1}=f_{2}=2 \lambda_{3}+\frac{1}{2} \lambda_{5}+\frac{1}{2} \lambda_{6}
\end{aligned}
$$

Another submatrix is defined by means of the states $\left(w_{1}^{+} w_{1}^{-}, w_{2}^{+} w_{2}^{-}, \frac{z_{1} z_{1}}{\sqrt{2}}, \frac{z_{2} z_{2}}{\sqrt{2}}, \frac{h_{1} h_{1}}{\sqrt{2}}, \frac{h_{2} h_{2}}{\sqrt{2}}\right)$; it reads

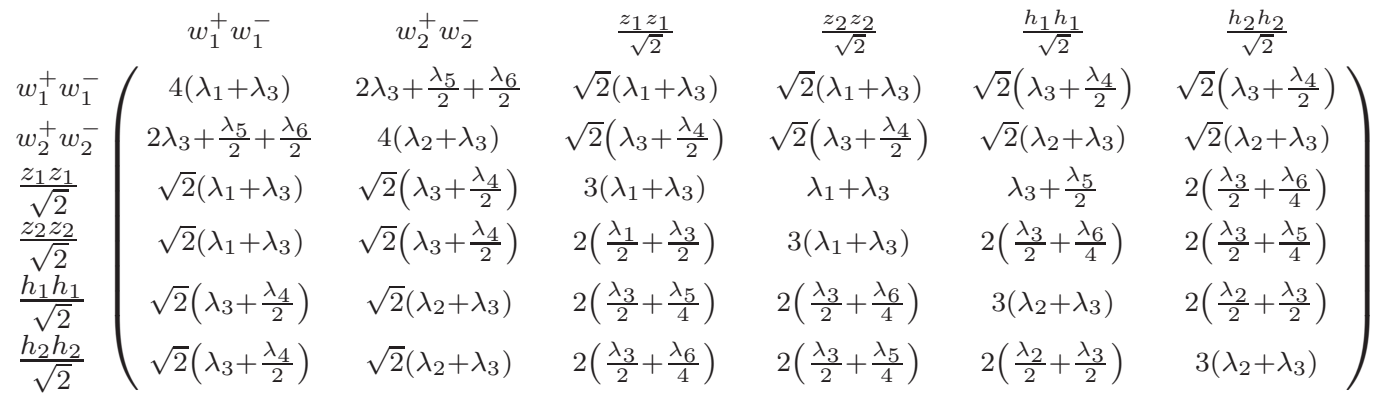

and its eigenvalues are

$$
\begin{aligned}
& a_{ \pm}=3\left(\lambda_{1}+\lambda_{2}+2 \lambda_{3}\right) \pm \sqrt{9\left(\lambda_{1}-\lambda_{2}\right)^{2}+\left[4 \lambda_{3}+\lambda_{4}+\frac{1}{2}\left(\lambda_{5}+\lambda_{5}\right)\right]^{2}} \\
& b_{ \pm}=\lambda_{1}+\lambda_{2}+2 \lambda_{3} \pm \sqrt{\left(\lambda_{1}-\lambda_{2}\right)^{2}+\frac{1}{4}\left(-2 \lambda_{4}+\lambda_{5}+\lambda_{6}\right)^{2}} \\
& c_{ \pm}=\lambda_{1}+\lambda_{2}+2 \lambda_{3} \pm \sqrt{\left(\lambda_{1}-\lambda_{2}\right)^{2}+\frac{1}{4}\left(\lambda_{5}-\lambda_{6}\right)^{2}}
\end{aligned}
$$

A third submatrix

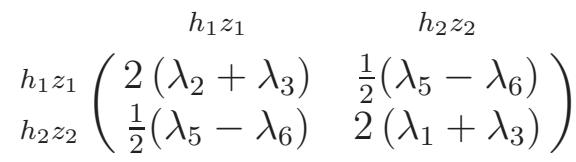


has eigenvalues $c_{ \pm}$(see (19)). Finally, there are submatrices corresponding to charged states $\left(h_{1} w_{1}^{+}, h_{2} w_{1}^{+}, z_{1} w_{1}^{+}, z_{2} w_{1}^{+}, h_{1} w_{2}^{+}, h_{2} w_{2}^{+}, z_{1} w_{2}^{+}, z_{2} w_{2}^{+}\right)$:

$$
\begin{aligned}
& \begin{array}{cccc}
h_{1} w_{1}^{+} & h_{2} w_{1}^{+} & z_{1} w_{1}^{+} & z_{2} w_{1}^{+} \\
h_{1} w_{1}^{+} \\
h_{2} w_{1}^{+} \\
z_{1} w_{1}^{+} \\
z_{2} w_{1}^{+}
\end{array}\left(\begin{array}{cccc}
2\left(\lambda_{1}+\lambda_{3}\right) & \frac{-\lambda_{4}}{2}+\frac{\lambda_{5}}{2} & 0 & \frac{i}{2} \lambda_{4}-\frac{i}{2} \lambda_{6} \\
\frac{-\lambda_{4}}{2}+\frac{\lambda_{5}}{2} & 2\left(\lambda_{2}+\lambda_{3}\right) & \frac{i}{2} \lambda_{4}-\frac{i}{2} \lambda_{6} & 0 \\
0 & \frac{-i}{2} \lambda_{4}+\frac{i}{2} \lambda_{6} & 2\left(\lambda_{1}+\lambda_{3}\right) & \frac{-\lambda_{4}}{2}+\frac{\lambda_{5}}{2} \\
\frac{-i}{2} \lambda_{4}+\frac{i}{2} \lambda_{6} & 0 & \frac{-\lambda_{4}}{2}+\frac{\lambda_{5}}{2} & 2\left(\lambda_{2}+\lambda_{3}\right)
\end{array}\right) \\
& h_{1} w_{2}^{+} \quad h_{2} w_{2}^{+} \quad z_{1} w_{2}^{+} \quad z_{2} w_{2}^{+} \\
& \begin{array}{l}
h_{1} w_{2}^{+} \\
h_{2} w_{2}^{+} \\
z_{1} w_{2}^{+} \\
z_{2} w_{2}^{+}
\end{array}\left(\begin{array}{cccc}
2\left(\lambda_{3}+\frac{\lambda_{4}}{2}\right) & \frac{-\lambda_{4}}{2}+\frac{\lambda_{5}}{2} & 0 & \frac{i}{2} \lambda_{4}-\frac{i}{2} \lambda_{6} \\
\frac{-\lambda_{4}}{2}+\frac{\lambda_{5}}{2} & 2\left(\lambda_{3}+\frac{\lambda_{4}}{2}\right) & \frac{i}{2} \lambda_{4}-\frac{i}{2} \lambda_{6} & 0 \\
0 & \frac{-i}{2} \lambda_{4}+\frac{i}{2} \lambda_{6} & 2\left(\lambda_{3}+\frac{\lambda_{4}}{2}\right) & \frac{-\lambda_{4}}{2}+\frac{\lambda_{5}}{2} \\
\frac{-i}{2} \lambda_{4}+\frac{i}{2} \lambda_{6} & 0 & \frac{-\lambda_{4}}{2}+\frac{\lambda_{5}}{2} & 2\left(\lambda_{3}+\frac{\lambda_{4}}{2}\right)
\end{array}\right)
\end{aligned}
$$

Their eigenvalues are the $f_{-}, e_{2}, f_{1}, c_{ \pm}, b_{ \pm}$shown above and, in addition,

$$
p_{1}=2\left(\lambda_{3}+\lambda_{4}\right)-\frac{1}{2} \lambda_{5}-\frac{1}{2} \lambda_{6}
$$

Unitarity conditions (15) for the eigenvalues listed above give the constraints

$$
\left|a_{ \pm}\right|,\left|b_{ \pm}\right|,\left|c_{ \pm}\right|,\left|f_{ \pm}\right|,\left|e_{1,2}\right|,\left|f_{1}\right|,\left|p_{1}\right| \leq 8 \pi
$$

Note that an independent derivation of these inequalities based on symmetries of the Higgs potential can be found in the papers [9, 10].

\section{Independent inequalities}

However, the inequalities (24) are not all independent. Indeed, it is not difficult to observe some simple relations as

$$
\begin{aligned}
3 f_{1} & =p_{1}+e_{1}+f_{+} \\
3 e_{2} & =2 p_{1}+e_{1} \\
3 f_{-} & =2 p_{1}+f_{+}
\end{aligned}
$$

and this means that the inequalities $\left|p_{1}\right|,\left|f_{+}\right|,\left|e_{1}\right| \leq 8 \pi$ imply $\left|f_{1}\right|,\left|e_{2}\right|,\left|f_{-}\right| \leq 8 \pi$. Further, the eigenvalues (19) in the remaining inequalities can be rewritten as

$$
\begin{aligned}
& a_{ \pm}=3 \lambda_{123} \pm \sqrt{\left(3 \lambda_{12}\right)^{2}+\frac{1}{4}\left(f_{+}+e_{1}+2 p_{1}\right)^{2}} \\
& b_{ \pm}=\lambda_{123} \pm \sqrt{\left(\lambda_{12}\right)^{2}+\frac{1}{36}\left(f_{+}+e_{1}-2 p_{1}\right)^{2}} \\
& c_{ \pm}=\lambda_{123} \pm \sqrt{\left(\lambda_{12}\right)^{2}+\frac{1}{36}\left(f_{+}-e_{1}\right)^{2}}
\end{aligned}
$$


where $\lambda_{123}=\lambda_{1}+\lambda_{2}+2 \lambda_{3}$ and $\lambda_{12}=\lambda_{1}-\lambda_{2}$. In the case $\lambda_{123}>0$ the inequalities for the $a_{-}, b_{-}, c_{-}$follow from $a_{+}, b_{+}, c_{+} \leq 8 \pi$. For $\lambda_{123}<0$ the situation is similar, with interchanges $(a, b, c)_{ \pm} \rightarrow(a, b, c)_{\mp}$ and $\lambda_{123} \rightarrow-\lambda_{123}$.

The authors [7] noticed that among the latter inequalities, the strongest one is $a_{+}<8 \pi$; Indeed, using (24) and (26) one can show that for $\lambda_{123}>0$ the remaining ones follow from it. In the case $\lambda_{123}<0$ the same statement is true concerning $a_{+}<8 \pi$.

Thus, it is sufficient to solve the inequalities

$$
\left|a_{ \pm}\right|,\left|f_{+}\right|,\left|e_{1}\right|,\left|p_{1}\right| \leq 8 \pi
$$

In fact, the inequality $a_{-}<8 \pi$ need not be taken into account in subsequent discussion; it turns out that this is weaker than the remaining ones and does not influence bounds in question (one can verify a posteriori that our solutions satisfy the constraints $a_{-}<8 \pi$ automatically).

\section{$5 \quad$ Upper bounds for $M_{A}$ and $M_{ \pm}$with $\xi=0$}

Before starting our calculation, let us recall that the condition $\xi=0$ means that the $Z_{2}$ symmetry-breaking parameter $\nu$ becomes $\nu=\lambda_{5}$ (see (3)). To proceed, we shall first fix convenient notations. The LQT bound for the SM Higgs mass sets a natural scale for our estimates, so let us introduce it explicitly:

$$
m_{\mathrm{LQT}}=\sqrt{\frac{4 \pi \sqrt{2}}{3 G_{\mathrm{F}}}}=\sqrt{\frac{8 \pi}{3}} v \doteq 712 \mathrm{GeV}
$$

(note that in writing eq.(28) we do not stick strictly to the original value [5], using rather the improved bound [15]). In the subsequent discussion we shall then work with the dimensionless ratios

$$
M=\frac{m}{m_{\mathrm{LQT}}}
$$

instead of the true scalar boson masses (denoted here generically as $m$ ). Further, an overall constant factor $16 \pi / 3$ can be absorbed in a convenient redefinition of the coupling constants, by writing

$$
\lambda_{i}^{\prime}=\frac{3 \lambda_{i}}{16 \pi}
$$

Finally, we introduce new variables

$$
X=M_{H}^{2}+M_{h}^{2}, \quad Y=M_{H}^{2}-M_{h}^{2}, \quad Z=\frac{\sin 2 \alpha}{\sin 2 \beta} Y
$$

that will help to streamline a bit the solution of the inequalities in question. 
Using equations (11) and the definitions shown above, the $\lambda^{\prime}$ can be expressed as

$$
\begin{aligned}
\lambda_{4}^{\prime} & =M_{ \pm}^{2} \\
\lambda_{6}^{\prime} & =M_{A}^{2} \\
\lambda_{3}^{\prime} & =\frac{1}{4} \frac{\sin 2 \alpha}{\sin 2 \beta} Y-\frac{1}{4} \lambda_{5}^{\prime}=\frac{Z}{4}-\frac{\lambda_{5}^{\prime}}{4} \\
\lambda_{12}^{\prime} & =\frac{1}{2 \sin ^{2} 2 \beta}\left[\left(X-2 \lambda_{5}^{\prime}\right) \cos 2 \beta-Y \cos 2 \alpha\right] \\
\lambda_{123}^{\prime} & =\frac{1}{2 \sin ^{2} 2 \beta}\left(X-Y \cos 2 \alpha \cos 2 \beta-2 \lambda_{5}^{\prime}\right)+\frac{\lambda_{5}^{\prime}}{2}
\end{aligned}
$$

Let us now discuss the possible bounds for the $M_{ \pm}, M_{A}$. These can be obtained from the inequalities for $\left|e_{1}\right|,\left|f_{+}\right|,\left|p_{1}\right|$, which read, in our new notation

$$
\begin{aligned}
\left|\frac{Z}{2}-\lambda_{5}^{\prime}-M_{ \pm}^{2}+\frac{5}{2} M_{A}^{2}\right| & \leq \frac{3}{2} \\
\left|\frac{Z}{2}+2 \lambda_{5}^{\prime}-M_{ \pm}^{2}-\frac{1}{2} M_{A}^{2}\right| & \leq \frac{3}{2} \\
\left|\frac{Z}{2}-\lambda_{5}^{\prime}+2 M_{ \pm}^{2}-\frac{1}{2} M_{A}^{2}\right| & \leq \frac{3}{2}
\end{aligned}
$$

The relations (33) are linear with respect to the $M_{ \pm}^{2}, M_{A}^{2}$ and one can thus view the domain defined by these inequalities as a hexagon in the plane $\left(M_{ \pm}^{2}, M_{A}^{2}\right)$. Then it is clear that the highest possible value of a mass variable in question will correspond to a vertex (or a whole hexagon side). By examining all possible cases one finds easily that for $M_{ \pm}^{2}$, such a "critical" vertex satisfies the condition $-f_{+}=p_{1}=8 \pi$; in view of (33) this means that it corresponds to the values

$$
\left(M_{ \pm}^{2}, M_{A}^{2}\right)=\left(1+\lambda_{5}^{\prime}, 1+Z+2 \lambda_{5}^{\prime}\right)
$$

Such a maximum value of the $M_{ \pm}^{2}$ is indeed formally admissible (in the sense that by reaching it one does not leave the parametric space of the considered model). To see this, one can substitute in eq. (34) $M_{A}^{2}=\lambda_{5}^{\prime}, M_{H}^{2}=1+\lambda_{5}^{\prime}, M_{h}^{2}=0, \alpha=\pi-\beta$. Thus, the bound becomes

$$
M_{ \pm}^{2} \leq 1+\lambda_{5}^{\prime}
$$

Similarly, for $M_{A}^{2}$ the extremal solution corresponds to a hexagon vertex defined by $e_{1}=$ $-f_{+}=8 \pi$ and its coordinates in the $\left(M_{ \pm}^{2}, M_{A}^{2}\right)$ plane are then

$$
\left(M_{ \pm}^{2}, M_{A}^{2}\right)=\left(1+\frac{Z}{2}+\frac{3}{2} \lambda_{5}^{\prime}, 1+\lambda_{5}^{\prime}\right)
$$

The parameter values that saturate this maximum are analogous and one has to take $M_{ \pm}^{2}=\lambda_{5}^{\prime} / 2, M_{H}^{2}=1+\lambda_{5}^{\prime}, M_{h}^{2}=0, \alpha=\pi-\beta$. In this way, the bound for $M_{A}^{2}$ becomes the same as that for the $M_{ \pm}^{2}$, namely

$$
M_{A}^{2} \leq 1+\lambda_{5}^{\prime}
$$




\section{Upper bounds for $M_{h}, M_{H}$ with $\xi=0$}

Let us now proceed to discuss the upper bounds for $M_{H}$ and $M_{h}$. If we considered the relevant constraints without any further specification of the scalar bosons $h$ and $H$, we would get the same result for both particles, since their interchange corresponds just to the replacement $\alpha \rightarrow-\alpha$ (cf. eq. (10)). Thus, let us add the condition $M_{h} \leq M_{H}$ (i.e. $Y>0$ ). In such a case, we will solve just the inequality $a_{+}<8 \pi$ (which puts the most stringent bounds on the variables $X, Y$ ) and in the obtained solution we will constrain the $M_{A}, M_{ \pm}$so as to satisfy the rest of the inequalities.

The basic constraint $a_{+}<8 \pi$ is quadratic with respect to the $X, Y$ and reads (cf. the expression (19))

$$
\sqrt{\left[(X-Y \cos 2 \alpha \cos 2 \beta)-\lambda_{5}^{\prime}\left(2-\sin ^{2} 2 \beta\right)+\right.}
$$

To work it out, we will employ the following trick: As a first step, we will consider a simpler inequality, which is obtained from (34) by discarding the second term under the square root; in other words, we will first assume that

$$
Y \frac{\sin 2 \alpha}{\sin 2 \beta}-\frac{\lambda_{5}^{\prime}}{2}+M_{ \pm}^{2}+\frac{M_{A}^{2}}{2}=0
$$

Of course, the "reduced" constraint

$$
X-Y \cos 2 \alpha \cos 2 \beta-\lambda_{5}^{\prime}\left(2-\sin ^{2} 2 \beta\right)+\left|X \cos 2 \beta-Y \cos 2 \alpha-2 \lambda_{5}^{\prime} \cos 2 \beta\right| \leq \sin ^{2} 2 \beta
$$

is in general weaker than the original one. Nevertheless, in a next step we will be able to show that the obtained mass bound does get saturated for appropriate values of the other parameters (such that the condition (39) is met) - i.e. that in this way we indeed get the desired minimum upper mass bound corresponding to the original constraint (38). Thus, let us examine the inequality (40). Obviously, we have to distinguish two possible cases:

1. $\left(X-2 \lambda_{5}^{\prime}\right) \cos 2 \beta \geq Y \cos 2 \alpha$.

Then one has

$$
X(1+\cos 2 \beta)-Y(1+\cos 2 \beta) \cos 2 \alpha-\lambda_{5}^{\prime}(1+\cos 2 \beta) \leq \sin ^{2} 2 \beta
$$

Making use of our assumption, we can get from (41) a simple constraint that does not involve $Y$, namely

$$
X \leq 1+\lambda_{5}^{\prime}
$$

(to arrive at the last relation, we had to divide by the factor $1-\cos 2 \beta$; when it vanishes, we can use directly the original inequality (38) and get the same result). 
2. $\left(X-2 \lambda_{5}^{\prime}\right) \cos 2 \beta \leq Y \cos 2 \alpha$.

In a similar way as in the preceding case, the inequality (40) implies the same bound (42).

Thus, having constrained $X=M_{H}^{2}+M_{h}^{2}$ according to (42), we can obviously also write

$$
M_{H}^{2} \leq 1+\lambda_{5}^{\prime}
$$

Now, it is not difficult to see that for $M_{h}^{2}=0, M_{ \pm}^{2}=\lambda_{5}^{\prime}+\frac{1}{2}, M_{A}^{2}=1+\lambda_{5}^{\prime}, \alpha=\pi-\beta$, eq. (39) is satisfied with $M_{H}^{2}=1+\lambda_{5}^{\prime}$ and means that (43) represents the mass upper bound pertinent to the original unitarity constraint (38).

The bound for the $M_{h}$ is obtained from (42) by using there our subsidiary condition $M_{h} \leq M_{H}$; one thus has

$$
M_{h}^{2} \leq \frac{1}{2}\left(1+\lambda_{5}^{\prime}\right)
$$

The upper limit in (44) gets saturated (i.e. $M_{h}^{2}=\frac{1}{2}\left(1+\lambda_{5}^{\prime}\right)$ ) for $M_{H}=M_{h}, M_{A}^{2}=0$, $M_{ \pm}^{2}=\lambda_{5}^{\prime} / 2, \alpha=3 \pi / 4, \beta=\pi / 4$. It is worth noticing that here we have fixed a particular value of the angle $\beta$, while all previous constraints were independent of $\beta$ (i.e. for any $\beta$ we were then able to find an appropriate value of $\alpha$ ). A more detailed analysis shows that, in general, the upper bound for the $M_{h}$ indeed depends explicitly on the $\beta$. To derive the corresponding formula, we consider the boundary value $M_{h}=M_{H}$ (i.e. $Y=0$ ) and use also eq. (391). The inequality (38) then becomes

$$
M_{h}^{2}-\lambda_{5}^{\prime}\left(1-\frac{\sin ^{2} 2 \beta}{2}\right)+\left|M_{h}^{2} \cos 2 \beta-\lambda_{5}^{\prime} \cos 2 \beta\right| \leq \frac{\sin ^{2} 2 \beta}{2}
$$

To work it out, we will assume that $M_{h}^{2} \geq \lambda_{5}^{\prime}$ (taking into account (44) this means $\lambda_{5}^{\prime} \leq 1$; in fact, one can do even without such a restriction, but for our perturbative treatment only sufficiently small values of the $\lambda_{5}^{\prime}$ are of real interest). The inequality (45) then becomes

$$
M_{h}^{2} \leq \frac{\left(1-\lambda_{5}^{\prime}\right)}{2} \frac{(1+\cos 2 \beta)(1-\cos 2 \beta)}{1+|\cos 2 \beta|}+\lambda_{5}^{\prime}
$$

Obviously, the maximum bound (44) is recovered from the last expression for $\beta=\pi / 4$. Let us also remark that the choice $\alpha=\pi-\beta$ comes, as in all previous cases, from the requirement $Z=-Y$.

\section{$7 \quad$ Upper bound for the lightest scalar for $\xi=0$}

One can notice that any Higgs mass upper limit discussed so far gets saturated only when at least one of the other scalar masses vanishes. Thus, another meaningful question arising in this connection is what can be an upper bound for the lightest Higgs boson (within a considered set of the five scalars $h, H, A^{0}, H^{ \pm}$). Let us first take $h$ to be the lightest scalar state; it means that in our analysis we will include the additional assumption 


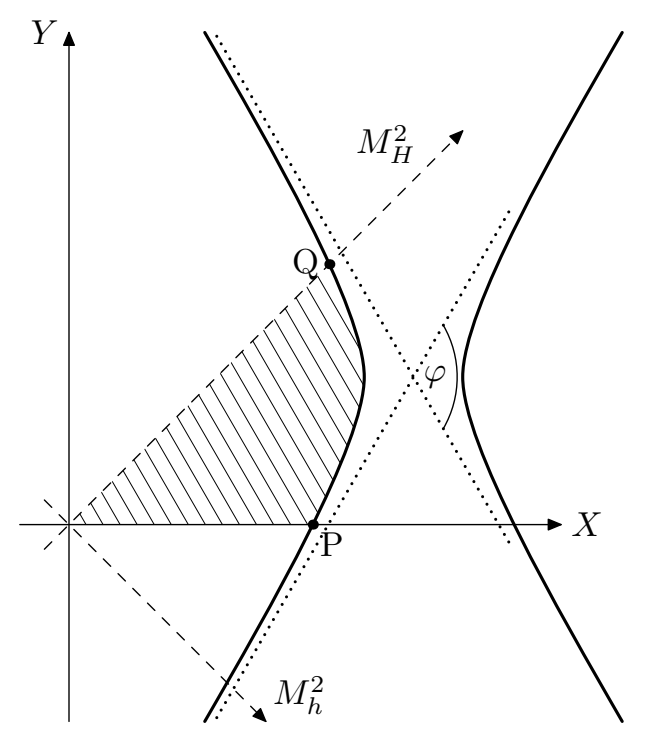

Figure 1: The region of admissible values of $M_{h}^{2}$ if $h$ is assumed to be the lightest scalar

$M_{h} \leq M_{H}, M_{A}, M_{ \pm}$. The procedure we are going to employ is a modest generalization of the earlier calculation [7. Squaring the inequality (38) one gets

$$
\left(X-X_{0}\right)^{2}-\left(1-\frac{5}{9} \sin ^{2} 2 \alpha\right)\left(Y-Y_{0}\right)^{2} \geq R^{2}
$$

where $X_{0}, Y_{0}$ and $R$ depend on $\lambda_{5}^{\prime}, \alpha, \beta, M_{ \pm}^{2}+M_{A}^{2} / 2$. This inequality defines the domain bounded by the hyperbola shown in Fig. 1] but the original constraint (38) corresponds just to its left-hand part. In order to find the solution, one should realize that the slope of the asymptote with respect to the $X$-axis must be greater than the slope of the straight lines $X= \pm Y$ (this follows from the fact that the coefficient $1-\frac{5}{9} \sin ^{2} 2 \alpha$, multiplying the $Y^{2}$ in (47), is less than one). Because of that, the maximum value of the $M_{h}$ corresponds to $Y=0$ and $a_{+}=8 \pi$, and we are thus led to the equation

$$
X-\lambda_{5}^{\prime}\left(2-\sin ^{2} 2 \beta\right)+\sqrt{\cos ^{2} 2 \beta\left(X-2 \lambda_{5}^{\prime}\right)^{2}+\frac{4}{9} \sin ^{4} 2 \beta\left(M_{ \pm}^{2}+\frac{M_{A}^{2}}{2}-\frac{\lambda_{5}^{\prime}}{2}\right)}=\sin ^{2} 2 \beta
$$

It is clear that for smaller $M_{ \pm}, M_{A}$ one has a bigger value of the $M_{h}$, so the needed upper estimate is obtained for $M_{ \pm}=M_{A}=M_{h}$ (note also that from $Y=0$ one has $\left.X=2 M_{h}^{2}\right)$. In this way one gets an equation for maximum $M_{h}$ :

$$
2 M_{h}^{2}-\lambda_{5}^{\prime}\left(2-\sin ^{2} 2 \beta\right)+\sqrt{4\left(M_{h}^{2}-\lambda_{5}^{\prime}\right)^{2} \cos ^{2} 2 \beta+\sin ^{4} 2 \beta\left(M_{h}^{2}-\frac{1}{3} \lambda_{5}^{\prime}\right)^{2}}=\sin ^{2} 2 \beta
$$

From eq. (49) one can calculate the $M_{h}^{2}$ as a function of $\sin ^{2} 2 \beta$. It can be shown that for $\lambda_{5}^{\prime}<3 / 5$ this function is increasing, i.e. the maximum is reached for $\beta=\pi / 4$ and its value 


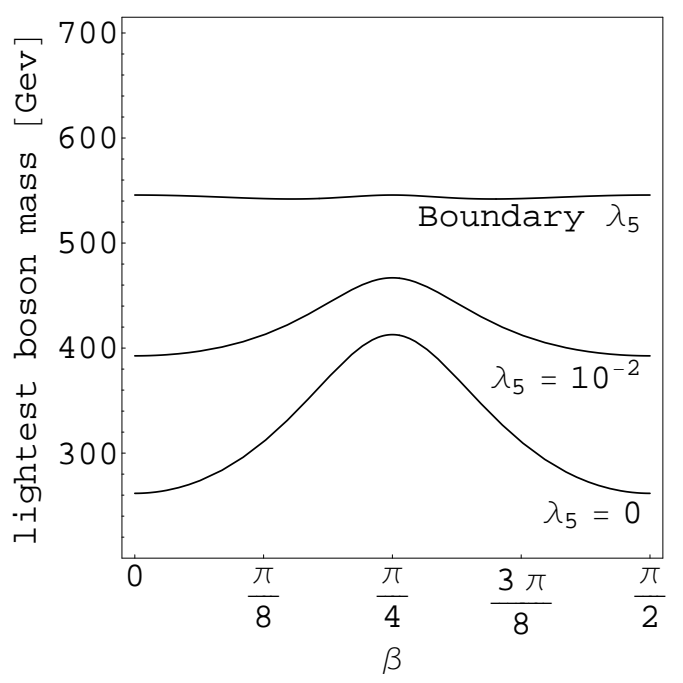

Figure 2: Dependence of lightest boson mass on $\beta$

becomes

$$
M_{h}^{2}=\frac{1}{3}+\frac{4}{9} \lambda_{5}^{\prime}
$$

We do not display the explicit dependence of the maximum $M_{h}$ on the $\beta$, but it is clear that the solution of eq. (49) is straightforward. Finally, we should also examine the cases where the lightest Higgs boson mass is either $M_{A}$ or $M_{ \pm}$. However, from the above discussion it is clear that both these extremes occur when $M_{h}=M_{A}=M_{ \pm}$.

Similarly, from eq. (48) one can derive a constraint for the mass of the lightest neutral scalar boson (which we denote $M_{n}$ ). In this case we substitute there $X=2 M_{n}^{2}, M_{A}^{2}=M_{n}^{2}$, $M_{ \pm}^{2}=0$ and obtain thus the equation

$$
2 M_{n}^{2}-\lambda_{5}^{\prime}\left(2-\sin ^{2} 2 \beta\right)+\sqrt{4\left(M_{n}^{2}-\lambda_{5}^{\prime}\right)^{2} \cos ^{2} 2 \beta+\sin ^{4} 2 \beta\left(\frac{M_{n}^{2}}{3}-\frac{1}{3} \lambda_{5}^{\prime}\right)^{2}}=\sin ^{2} 2 \beta
$$

From eq. (51) one then obtains the $M_{n}^{2}$ as a function of $\sin ^{2} 2 \beta$, which is increasing for $\lambda_{5}^{\prime}<1$. Its maximum reached at $\beta=\pi / 4$ becomes

$$
M_{n}^{2}=\frac{3}{7}+\frac{4}{7} \lambda_{5}^{\prime}
$$

\section{Numerical solution for $\xi \neq 0$}

In the general case with $\xi \neq 0$ (i.e. with $C P$ violation in the scalar sector) we have not been able to solve the inequalities (27) analytically, so we had to resort to an appropriate numerical procedure. The main result we have obtained in this way is that for small values 
of the parameter $\nu$ (see eq. (3)), in particular for $\nu^{\prime} \in\langle 0,0.3\rangle$, the upper mass bounds in question are the same as for $\xi=0$. The interval has been chosen such that the variations in the upper estimates be at the level of $50-100 \%$, the validity of our theoretical estimates is guaranteed up to $\nu^{\prime}<3 / 5$ (see the remark below eq. (49)).

Our numerical procedure consists in solving the inequalities (27) on the space of parameters $\lambda_{1,2,3,4,5,6}^{\prime}$ and $\xi$ restricted by the condition (3), where one also adds constraints for the existence of a minimum of the potential (11): $\lambda_{4}^{\prime}>0$ (i.e. $m_{ \pm}^{2}>0$, see (11)) and the requirement of positive definiteness of the matrix (9) (i.e. $m_{A, H, h}^{2}>0$ ). On this parametric subspace we have looked for the maximum values of the following quantities:

1. Mass of the charged Higgs boson $m_{ \pm}$(see Fig. 3)

2. Mass of the lightest Higgs boson (see Fig. 4)

3. Mass of the lightest neutral Higgs, i.e. the lightest one among the $A, H, h$ (see Fig. (5))

4. Mass of the heaviest neutral Higgs, i.e. the heaviest among the $A, H, h$ (see Fig. 6).

Let us remark that in this case we have not distinguished between $A$ and $h, H$, which are superpositions of the $C P$-odd and $C P$-even states.

In our plots we display, apart from the dependence of masses in question on the $\nu$, also the values of the parameter $\xi$ in the case $\lambda_{5}=\lambda_{6}$ and $\lambda_{5} \neq \lambda_{6}$ respectively, in order to be able to distinguish the extreme cases without $C P$ violation $\left(\xi=k \pi / 2\right.$ or $\lambda_{5}=\lambda_{6}$, see the discussion in Section 21). From Figs. 3, 4, 5, 6] it can be seen that all examined mass upper bounds are reached just in the aforementioned extreme cases. In view of this, we can make use of our previous analytic expressions, except for the case 3. which we have not solved analytically.

Our results have been simulated by means of the computer program Matlab 6.0, package optim, with the help of the function fmincon. The numerical errors are mostly due to an insufficiently smooth condition for the positive definiteness of the matrix (9).

\section{Conclusions}

In the present paper we have reconsidered upper bounds for the scalar boson masses within THDM, by using the well-known technical constraint of tree-level unitarity. Our analysis should extend and generalize the results of some previous treatments, in particular those obtained in the papers [8] and [7]. Although we basically employ the traditional methods, we have tried to present some details of the calculations not shown in the earlier papers we have done so not only for the reader's convenience, but also to provide a better insight into the origin of the numerical results displayed here. As we have already noted in the Introduction, some new relevant papers on the subject have appeared quite recently (see [9, 10, 11]). In these works, the structure of the unitarity constraints is discussed in detail within a rather general THDM, but there is no substantial overlap with our results, since our main point is rather a detailed explicit solution of the inequalities in question. 

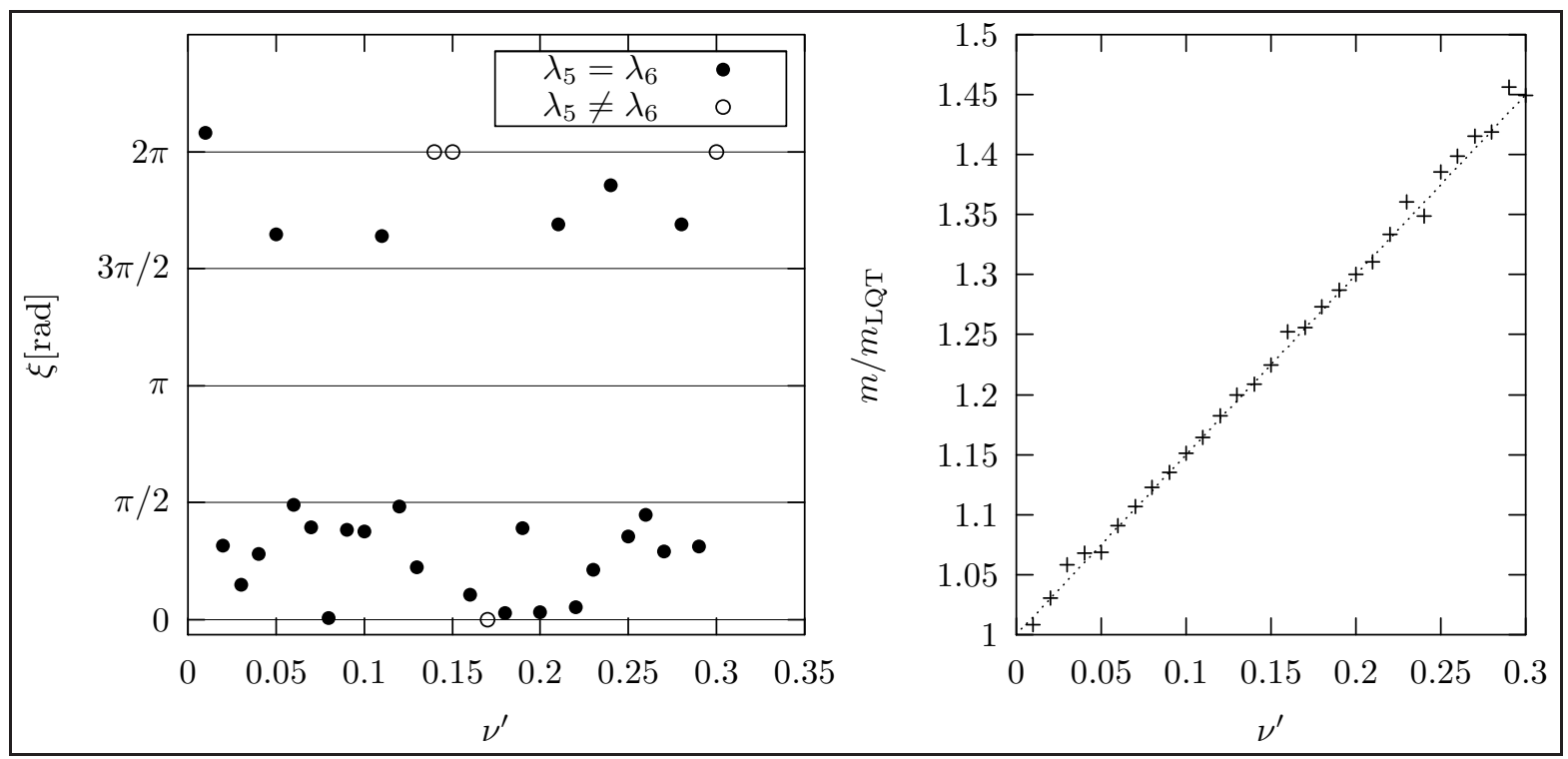

Figure 3: Charged Higgs boson, with theoretical estimate (35)

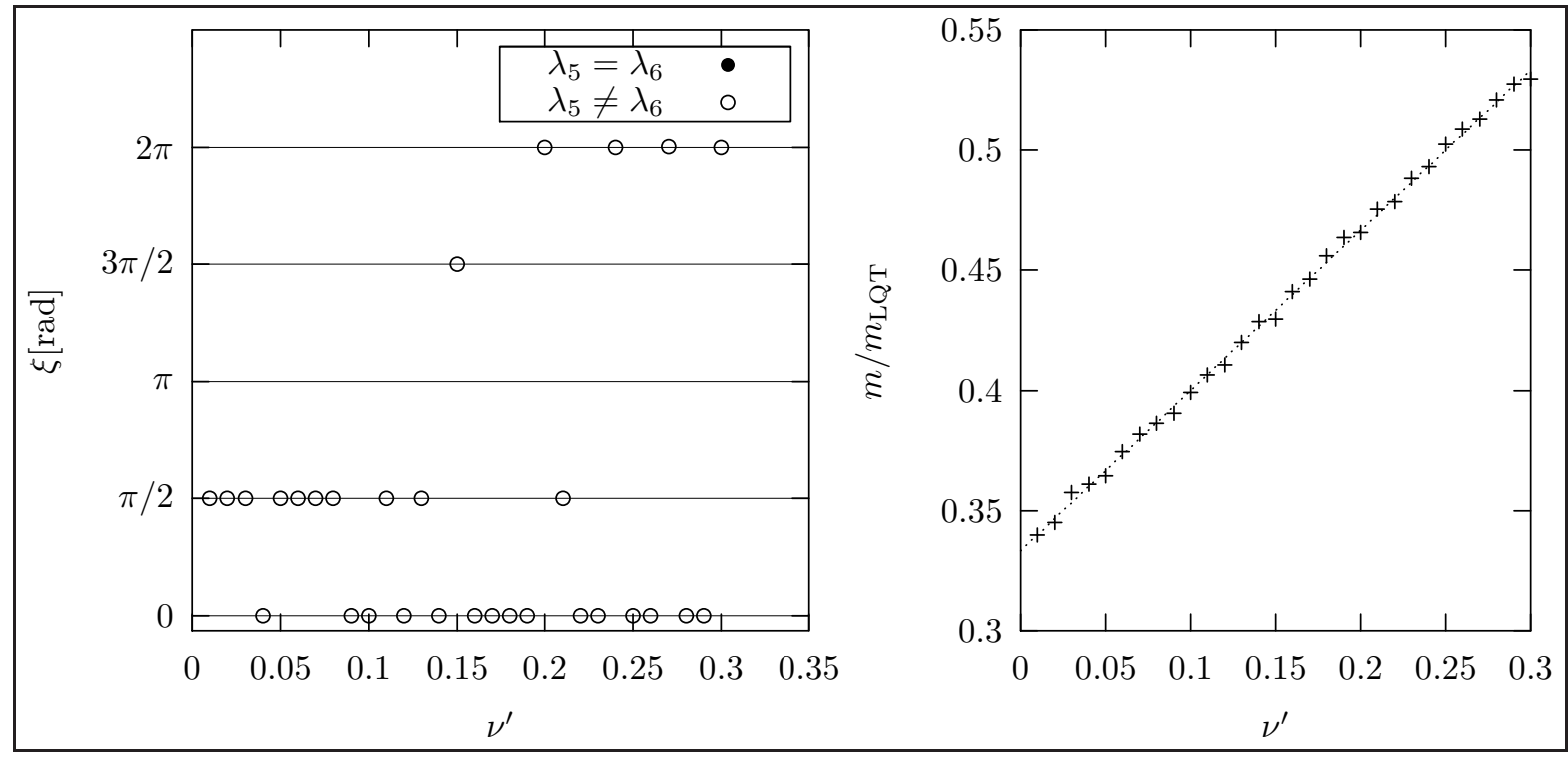

Figure 4: Lightest scalar mass, with theoretical estimate (50) 

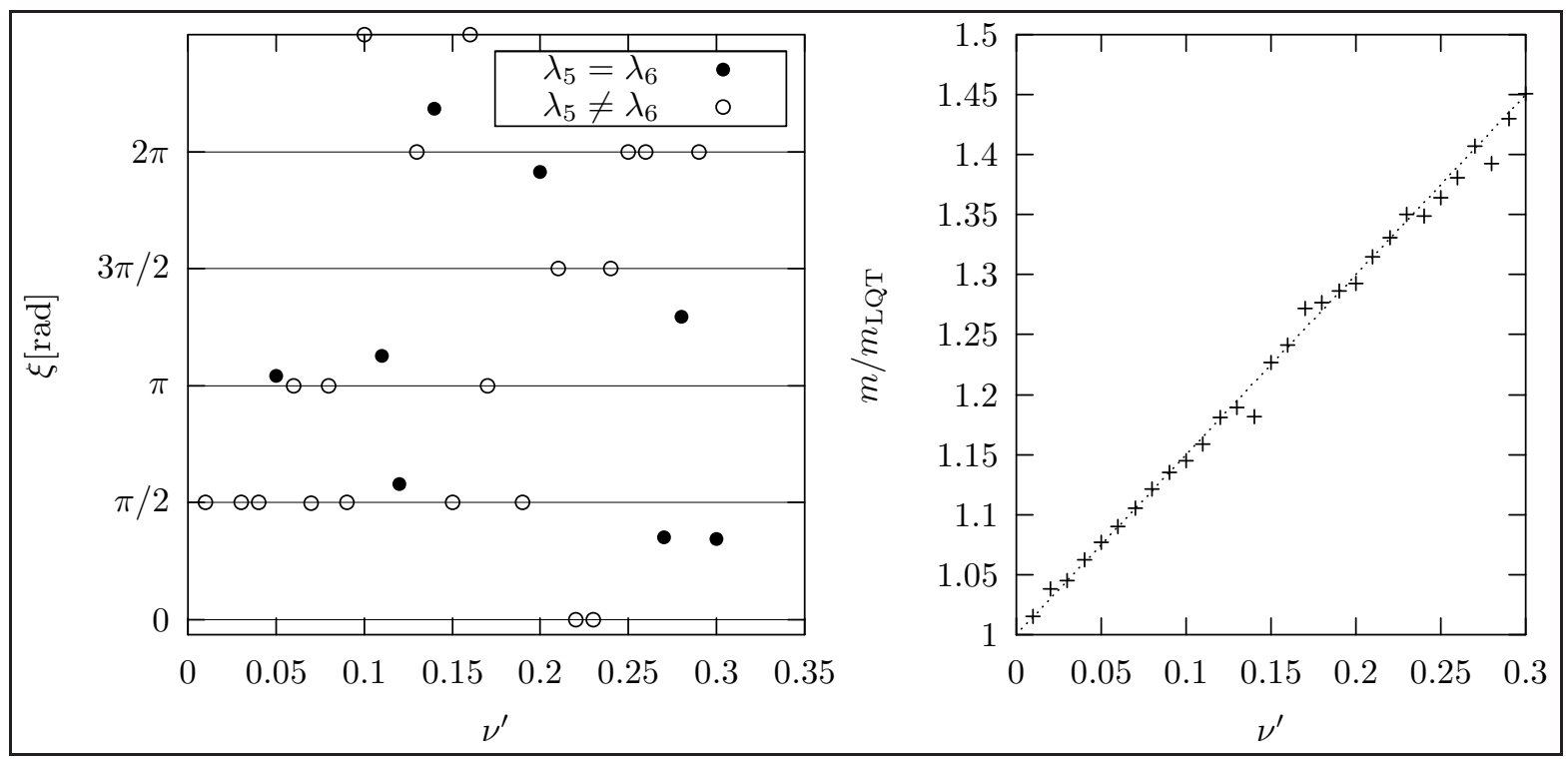

Figure 5: Heaviest neutral Higgs boson, with theoretical estimate (43) or (37)

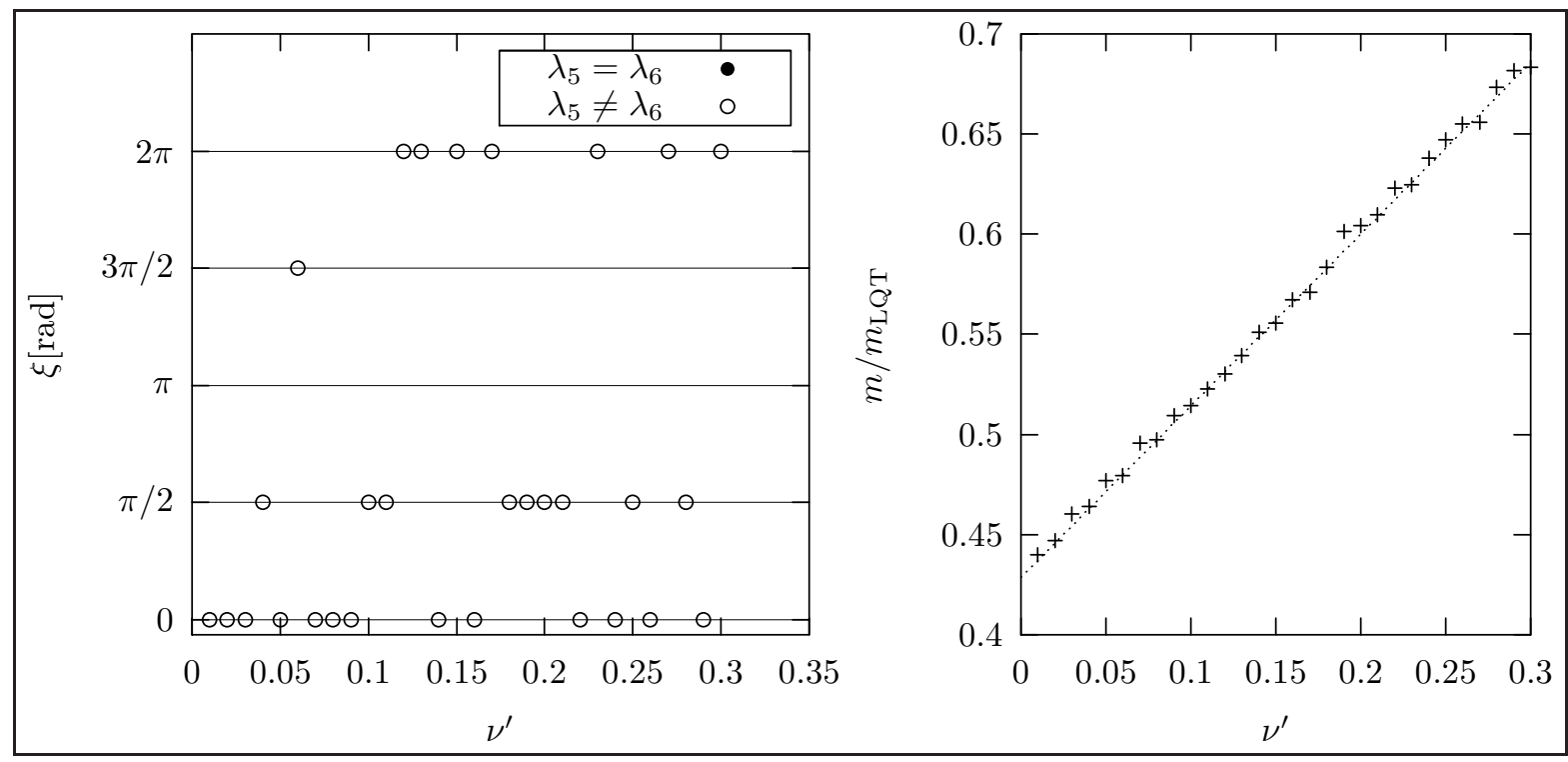

Figure 6: Lightest neutral boson with theoretical estimate (52) 
So, let us now summarize briefly our main results. We have found upper limits for Higgs boson masses in dependence on the parameter $\nu$ that embodies an information about possible flavour-changing neutral scalar-mediated interactions. The upper bounds are seen to grow with increasing $\nu$ (see Tab 1). On the other hand, this parameter cannot take on large values (to avoid a conflict with current phenomenology), and thus it makes no real sense to consider the mass estimates for an arbitrary $\nu$; in the present paper we restrict ourselves to $\nu \leq 0.4$ (cf. the condition used when deriving the relation (501)). In the case with no $C P$ violation in the scalar sector $(\xi=0)$, the relevant results are obtained from the inequalities (35), (37), (43), (44), and the bound for the lightest scalar is shown in eq. (50) (where one should also pass from $\lambda_{5}^{\prime}$ to $\lambda_{5}$ according to (30)). In Section 8 we have then verified that in the $C P$-violating case these values remain the same. The results are shown in Tab. 1. where we have singled out the case $\nu=0$ that corresponds to the absence of flavour-changing scalar currents. Let us remark that in the $C P$-violating case we do not distinguish between the $H$ and $A$, and in the $C P$-conserving case the bounds for $H$ and $A$ are the same.

Further, we have calculated an explicit dependence of the upper limit for the $M_{h}$ on the angle $\beta$ in the case with $\xi=0$. The analytic expression reads

$$
M_{h}^{2} \leq \frac{\sin ^{2} 2 \beta}{1+|\cos 2 \beta|}\left(\frac{1}{2}-\frac{3}{32 \pi} \lambda_{5}\right)+\lambda_{5} \frac{3}{16 \pi}
$$

(cf. (46) with the $\lambda_{5}$ retrieved). The dependence of the relevant bound for a lightest scalar boson can be obtained from eq. (49) and the results for some particular values of the $\lambda_{5}$ are depicted in Fig.2.

For $\nu=0$ and $\xi=0$, our results can be compared directly with those published in [7]. We get somewhat stronger bounds for $m_{A}$ and $m_{ \pm}$since, in addition to the set of constraints utilized in [7], we have employed also the inequality $p_{1}<8 \pi$, which stems from charged processes (cf. the end of Section 4) not considered in [7. On the other hand, our estimates for $m_{H}, m_{h}$ and the lightest scalar coincide with the results [7], since the above-mentioned extra inequality is not used here. It is also noteworthy that the upper limits for $m_{h}$ and $m_{H}$ coincide with the SM LQT bound if they are estimated separately and, depending on the number of the simultaneously estimated Higgs scalars, the coefficient 1/2 appears when we take two of them and $1 / 3$ when all of them are considered.

In the case $\xi=0$ and $\lambda_{5} \neq 0$ comparison with [8] is possible. Here we can compare only the corresponding numerical values, which turn out to be approximately equal when $\lambda_{5}=0$. However, for $\lambda_{5}=0$ our results obviously differ from those of [8]: in particular, the bounds for $m_{A}, m_{ \pm}$displayed in [8] appear to decrease with increasing $\lambda_{5}$. The authors [8] state that they used some fixed values of the angle $\beta$; for the purpose of a better comparison we have therefore calculated the $\beta$-dependence of the upper bound for $m_{h}$, with the result shown in (53). As it turns out, the $m_{A}$ and $m_{ \pm}$do not depend on $\beta$ in this case.

Finally, let us mention that in the $C P$-violating case we have not been able to get analytic results; we have only shown, numerically, that the maximum values of the masses in question are obtained for $\xi=0$, i.e. the upper mass bounds are the same as in the case with no $C P$ violation in the scalar sector. 


\begin{tabular}{|l|l|l|l|l|l|}
\hline & $\mathbf{H}$ & $\mathrm{A}$ & $\mathbf{H}^{ \pm}$ & $\mathrm{h}$ & lightest boson \\
\hline
\end{tabular}

Our results

\begin{tabular}{|l|c|c|c|}
\hline$m / m_{\mathrm{LQT}}$ & $\sqrt{1+\nu \frac{3}{16 \pi}}$ & $\sqrt{\frac{1}{2}+\nu \frac{3}{32 \pi}}$ & $\sqrt{\frac{1}{3}+\nu \frac{1}{12 \pi}}$ \\
\hline$m[\mathrm{GeV}]$ & $712 \mathrm{GeV}$ & $503 \mathrm{GeV}$ & $411 \mathrm{GeV}$ \\
\hline
\end{tabular}

Results [7]

\begin{tabular}{|c|c|c|c|c|c|}
\hline$m / m_{\mathrm{LQT}}$ & 1 & $\sqrt{3}$ & $\sqrt{\frac{3}{2}}$ & $\frac{1}{\sqrt{2}}$ & $\frac{1}{\sqrt{3}}$ \\
\hline$m[\mathrm{GeV}]$ & $712 \mathrm{GeV}$ & $1233 \mathrm{GeV}$ & $872 \mathrm{GeV}$ & $503 \mathrm{GeV}$ & $411 \mathrm{GeV}$ \\
\hline
\end{tabular}

Results [8]

\begin{tabular}{|l|l|l|l|l|l|}
\hline$m[\mathrm{GeV}]$ & $638 \mathrm{GeV}$ & $691 \mathrm{GeV}$ & $695 \mathrm{GeV}$ & $435 \mathrm{GeV}$ & - \\
\hline
\end{tabular}

Table 1: Comparison with other works

\section{References}

[1] T. D. Lee, Phys. Rev. D8, 1226 (1973).

[2] M. Sher, Phys. Rept. 179, 273 (1989).

[3] J. F. Gunion, H. E. Haber, G. L. Kane, and S. Dawson, The Higgs Hunter's Guide (Perseus Publishing, Cambridge, Massachusetts 2000).

[4] J. Abdallah et al. (DELPHI Collaboration), Eur. Phys. J. C34, 399 (2004) arXiv:hep-ex/0404012;

P. Achard et al. (L3 Collaboration), Phys. Lett. B583, 14 (2004) arXiv:hep-ex/0402003;

G. Abbiendi et al. (OPAL Collaboration), Eur. Phys. J. C40, 317 (2005) arXiv:hep-ex/0408097.

[5] B. W. Lee, C. Quigg, and H. B. Thacker, Phys. Rev. D16, 1519 (1977). 
[6] J. Maalampi, J. Sirkka, and I. Vilja, Phys. Lett. B265, 371 (1991);

R. Casalbuoni, D. Dominici, R. Gatto, and C. Giunti, Phys. Lett. B178, 235 (1986);

R. Casalbuoni, D. Dominici, F. Feruglio, and R. Gatto, Nucl. Phys. B299, 117 (1988);

H. Hüffel and G. Pócsik, Z. Phys. C8, 13 (1981).

[7] S. Kanemura, T. Kubota, and E. Takasugi, Phys. Lett. B313, 155 (1993) arXiv:hep-ph/9303263.

[8] A. G. Akeroyd, A. Arhrib, and E.-M. Naimi, Phys. Lett. B490, 119 (2000) arXiv:hep-ph/0006035.

[9] I. F. Ginzburg and I. P. Ivanov, arXiv:hep-ph/0312374.

[10] I. F. Ginzburg and M. Krawczyk, arXiv:hep-ph/0408011.

[11] I. F. Ginzburg and I. P. Ivanov, arXiv:hep-ph/0508020.

[12] M. Kladiva, Theoretical upper bounds for Higgs boson masses, Master's thesis, Charles University, Prague, 2003.

[13] H. Georgi, Hadronic J. 1, 155 (1978).

[14] S. L. Glashow and S. Weinberg, Phys. Rev. D15, 1958 (1977).

[15] W. J. Marciano, G. Valencia, and S. Willenbrock, Phys. Rev. D40, 1725 (1989).

[16] C. E. Vayonakis, Nuovo Cim. Lett. 17, 383 (1976);

M. S. Chanowitz and M. K. Gaillard, Nucl. Phys. B261, 379 (1985);

G. J. Gounaris, R. Kögerler, and H. Neufeld, Phys. Rev. D34, 3257 (1986). 